\title{
Telmisartan improves endothelial dysfunction and renal autoregulation in Dahl salt-sensitive rats
}

\author{
Minoru Satoh, Yoshisuke Haruna, Sohachi Fujimoto, Tamaki Sasaki and Naoki Kashihara
}

Hypertensive vascular disorders are characterized by endothelial dysfunction. Loss of renal autoregulation causes glomerular hypertension. However, the relationship between the autoregulatory response and glomerular damage has not been well examined. We examined the contributions of uncoupled endothelial nitric oxide synthase (eNOS) in hypertensive renal disease, and the relationship between the degree of autoregulation impairment and glomerular injury. We also investigated the effects of telmisartan on eNOS coupling and renal autoregulation. Male Dahl salt-sensitive hypertensive (DS) rats (14-week old) fed an $8 \%$ salt diet were used to examine endothelial dysfunction and impaired renal autoregulation caused by glomerular hypertension. Some DS rats were treated with telmisartan $\left(3.0 \mathrm{mg} \mathrm{kg}^{-1} \mathrm{day}^{-1}\right)$, an angiotensin receptor blocker, for 2 weeks. Increased superoxide production and decreased nitric oxide production, as detected by fluorescent indicator perfusion methods, were observed in the glomeruli and arterioles of hypertensive DS rats. Telmisartan improved the imbalance of superoxide and nitric oxide in the glomeruli and arterioles. Decreased serum tetrahydrobiopterin levels and coupled eNOS seen in the DS rat kidney were improved with telmisartan treatment. The endothelial relaxation reaction was impaired in DS rat aortic arteries.

Autoregulatory capacity in response to step changes in perfusion pressure was also impaired in DS rat kidney. Treatment with telmisartan improved these abnormalities. Endothelial dysfunction in the glomeruli and impaired renal autoregulation, which may cause glomerular sclerosis, were observed in DS rat kidney. Telmisartan treatment improves these dysfunctions in hypertensive renal disease.

Hypertension Research (2010) 33, 135-142; doi:10.1038/hr.2009.190; published online 20 November 2009

Keywords: angiotensin receptor blocker; eNOS uncoupling; nitric oxide; superoxide

\section{INTRODUCTION}

Hypertension is a well-known complication in chronic kidney disease and, at the same time, is one of the important risk factors for the development of cardiovascular disease and for the progression of renal insufficiency. ${ }^{1,2}$ Indeed, various studies have indicated that subjects with hypertension are at risk of developing end-stage renal disease. ${ }^{3-5}$ Hypertensive vascular disorders are characterized by pathological changes in endothelial cells in the early stage of hypertension, and endothelial cell dysfunction causes an imbalance between reactive oxygen species (ROS) and nitric oxide (NO) production. ${ }^{6}$ There are several overlapping mechanisms for endothelial dysfunction, including NO trapping by free radicals and reduced endothelial NO synthase (eNOS) activity. The eNOS is a homodimeric enzyme that generates $\mathrm{NO}$ and L-citrulline from L-arginine (L-Arg). However, in certain circumstances, eNOS may cause uncoupling that generates superoxide. ${ }^{7}$ Recent studies report the involvement of uncoupled eNOS during increased oxidative stress and endothelial dysfunction in association with atherosclerosis ${ }^{8}$ and diabetes. ${ }^{9}$ Evidence also suggests that the absence of the substrate L-Arg or cofactor tetrahydrobiopterin (BH4) uncouples eNOS, thereby producing superoxide rather than $\mathrm{NO},{ }^{10-12}$ which may contribute to the pathogenesis of endothelial dysfunction in arteries exposed to hypertension. However, little is known about the contributions of uncoupled eNOS in the context of hypertensive glomerular injury.

Pressure-mediated autoregulatory behavior operates by recognizing a change in renal perfusion pressure and initiating induction of autoregulatory resistance changes intended to stabilize renal blood flow. ${ }^{13}$ The net result is that renal blood flow and glomerular filtration rates remain relatively stable over a wide range of renal perfusion pressures. Autoregulatory behavior is significantly less efficient in pathological settings, such as in diabetes mellitus and in some forms of hypertension. Loss of autoregulatory efficiency could be one contributing factor, which leads to glomerular stress, glomerular compensation and perhaps glomerular injury and failure. Evidence indicates that renal autoregulation is impaired in some experimental animal models of hypertension. ${ }^{14,15}$ However, the relationship between the degree of autoregulation impairment and glomerular injury have not been well examined, and it is unclear whether blockade of renal autoregulation impairment would effectively inhibit hypertensive renal injury.

In this study, we examined whether uncoupled eNOS, which is a marker of endothelial dysfunction, and loss of renal autoregulation 
occur during hypertensive glomerular injury. We used Dahl salt-sensitive hypertensive (DS) rats to examine the NOS coupling statement in the kidney using the dihydroethidium (DHE) assay ${ }^{16}$ and in vivo $\mathrm{NO}$ and ROS imaging, ${ }^{17}$ and examined autoregulation capacity by measuring renal microvascular responses to step changes in renal perfusion pressure. Moreover, to elucidate whether angiotensin receptor blockade (ARB) has a beneficial effect against eNOS uncoupling and renal autoregulation, we examined DS rats treated with telmisartan, an angiotensin receptor blocker.

\section{METHODS}

\section{Experimental protocol}

Male DS rats (8-week old) were purchased from Clea Japan (Osaka, Japan). Animals were housed in a temperature- and humidity-controlled room with a 12:12 h light-dark cycle, and were fed standard laboratory animal chow with free access to tap water. In the first 6 weeks, rats were fed an $8 \%$ salt diet to induce hypertension. Systolic blood pressure (SBP) was also measured weekly in each rat using the tail-cuff method using an automatic sphygmomanometer (BP98A; Softron, Tokyo, Japan). After confirming the induction of hypertension, rats were randomly divided into three groups: the DS-H group $(n=15)$ consisting of untreated DS rats; the DS-H-Hyd group ( $n=15)$ consisting of DS rats treated with hydralazine (5.0 mg per rat per day); and the DS-H-Tel group $(n=15)$ consisting of DS rats treated with telmisartan $\left(3.0 \mathrm{mg} \mathrm{kg}^{-1} \mathrm{day}^{-1}\right)$. As a control, we used another group of DS normotensive rats fed a $0.9 \%$ salt diet (DS-L, $n=8$ ). The experimental protocol (No. 07-060) was approved in advance by the Ethics Review Committee for Animal Experimentation of Kawasaki Medical School, Kurashiki, Japan.

During the experimental period, body weight was measured weekly for each rat. All rats were killed after 2 weeks of treatment. Serum creatinine and blood urea nitrogen levels were measured after killing the rats. Before killing, 24-h urine samples were collected after placing the rats in metabolic cages and urine protein concentrations were measured spectrophotometrically using the Bradford method (Bio-Rad Protein Assay; Bio-Rad Laboratories, Tokyo, Japan).

\section{Glomerular damage score}

Half of the left kidneys were preserved in $4 \%$ paraformaldehyde for light microscopy. Paraffin-embedded tissue samples were cut into $4 \mu \mathrm{m}$-thick sections and stained with periodic acid-Schiff for histological studies. In each animal, 100 glomeruli were evaluated for the presence of sclerotic lesions. The severity of glomerular lesions was graded with a score of $0-3$ based on the percentage of glomerular involvement, as described previously. ${ }^{18}$

\section{Fluorescence spectrometric assay of $\mathrm{O}_{2-}$ production} in the renal cortex

To assess the source of ROS production, we performed fluorescence spectrometric assays. Fluorescence spectrometry of tissue $\mathrm{O}_{2}$ - production was carried out using the fluorogenic oxidation of DHE to ethidium as a measure of $\mathrm{O}_{2^{-}}$, as previously described. ${ }^{16}$ The renal cortex of the right kidney obtained from each rat was cut into small pieces, and the glomeruli were isolated by the mechanical graded sieving technique. After isolation, the purity of the final suspension was determined by light microscopic examination. On average, tubular contamination was $<5 \%$. Isolated glomeruli were homogenized in ice-cold 4-(2-hydroxyethyl)-1-piperazineethanesulfonic acid (HEPES) buffer containing $25 \mathrm{~mm}$ HEPES, $1 \mathrm{~mm}$ ethylenediaminetetraacetic acid (EDTA), and $0.1 \mathrm{~mm}$ phenylmethylsulfonyl fluoride. After centrifugation of the homogenate, the supernatant was used for this DHE assay. A part of the supernatant was used for western blotting. Enzyme activities of different pathways are expressed relative to the control. Following substrates or inhibitors were used in this study: nicotinamide adenine dinucleotide phosphate (NADPH) $(0.1 \mathrm{~mm})$ was used as a substrate for NADPH oxidases; $\mathrm{L}-\mathrm{Arg}(1 \mathrm{mM})$ was used as a substrate for NOS; NG-nitro-L-Arg methyl ester (1 mM) was used to block NOS activity; succinate $(5 \mathrm{mM})$ was used as a substrate for intramitochondrial $\mathrm{O}_{2^{-}}$production; antimycin $(0.05 \mathrm{~mm})$ was used to block the normal reaction in the respiratory chain; and xanthine $(0.1 \mathrm{~mm})$ was used as a substrate for xanthine oxidase. The effect of $\mathrm{BH} 4(0.01 \mathrm{mM})$ on $\mathrm{L}-\mathrm{Arg}$-induced $\mathrm{O}_{2}$ - production was also examined.

\section{In situ detection of NO and ROS}

To visualize the endothelial production levels of $\mathrm{NO}$ and ROS resulting from NOS coupling, in situ NO and ROS were imaged by confocal laser microscopy after perfusion with fluorescence indicators, as previously described. ${ }^{17}$ In brief, an 18-gauge needle connected to an infusion pump was inserted in the left ventricle. After the right atrium was cut, the whole body was perfused with PBS maintained at $37^{\circ} \mathrm{C}$. After exsanguination, the whole body was perfused with diaminorhodamine-4 $\mathrm{M}$ acetoxymethyl ester (DAR-4M AM; Daiichi Pure Chemicals, Tokyo, Japan) and $2^{\prime}, 7^{\prime}$-dichlorodihydrofluorescein-diacetate (DCFH-DA; Invitrogen, Tokyo, Japan) with $\mathrm{L}-\mathrm{Arg}$ and $\mathrm{CaCl}_{2}$. All unreacted DAR-4 $\mathrm{M}$ AM and DCFH-DA were removed by postperfusion with PBS. After fixation with $4 \%$ paraformaldehyde perfusion, the tissues were cut into $1 \mathrm{~mm}$ thick sections and placed on a slide glass. Fluorescent images of NO and ROS were obtained using confocal laser-scanning microscopy (TCS-NT, LeicaMicrosystems Japan, Tokyo, Japan). The mean NO and ROS fluorescence intensity of glomeruli (total of 100 glomeruli from five rats in each group) was analyzed using Leica TCS-NT system software (Leica-Microsystems).

\section{Western immunoblotting}

Western blots were performed to quantify total eNOS and its active dimer. Portions of the glomeruli samples ( $50 \mu \mathrm{g}$ per lane) were subjected to SDSPAGE. For immunoblot analysis of total eNOS, the samples were heated at $95^{\circ} \mathrm{C}$ for $5 \mathrm{~min}$ before electrophoresis. For immunoblot analysis of the dimeric form of eNOS, samples were not heated, and the temperature of the gel was maintained below $15{ }^{\circ} \mathrm{C}$ during electrophoresis (low-temperature SDSPAGE). ${ }^{17}$ Primary antibody $0.1 \mu \mathrm{g} / \mathrm{ml}$ rabbit anti-eNOS polyclonal antibody (Santa Cruz Biotechnology, Santa Cruz, CA, USA) was applied for $180 \mathrm{~min}$ at room temperature. The antibody was visualized using an enhanced chemiluminescence method (ECL plus; GE Healthcare Japan, Tokyo, Japan). The integrated density (density 3 area) of the bands was quantified using Image-J software (http://rsbweb.nih.gov/ij/).

\section{Determination of serum hydrobiopterin concentrations}

$\mathrm{BH} 4$ and dihydrobiopterin (BH2) concentrations were determined by highperformance liquid chromatography, as described previously. ${ }^{17}$ Serum samples were mixed at a ratio of $1: 1$ with a solution of $0.5 \mathrm{M}$ perchloric acid containing $0.1 \mathrm{~mm} \mathrm{Na} 2$-ethylenediaminetetraacetic acid and $0.1 \mathrm{mM} \mathrm{Na}_{2} \mathrm{~S}_{2} \mathrm{O}_{3}$ for protein separation. After filtration, $\mathrm{BH} 4$ concentrations in the samples were measured by HPLC. Detection of $\mathrm{BH} 4$ was carried out fluorometrically at wavelengths of $350 \mathrm{~nm}$ for excitation and of $440 \mathrm{~nm}$ for emission by postcolumn $\mathrm{NaNO}_{2}$ oxidation using a reversed-phase ion-pair LC system (LC-10 series; Shimadzu, Kyoto, Japan).

\section{Real-time quantitative PCR}

The mRNA expression of guanosine-5'-triphosphate cyclohydrolase 1 (GTPCH1), which is the rate-limiting enzyme in BH4 synthesis, and purinergic receptor P2X, ligand-gated ion channel 1 (P2rxl) were determined by real-time quantitative PCR. RNA isolation and real-time quantitative PCR for GTPCH1 and P2rxl were performed using the ABI Prism 7700 sequence detection system (Applied Biosystems, Foster City, CA, USA), as described previously. ${ }^{19}$ Primers and probes for P2rx1 (NM_012997) were as follows: rat P2rx1 forward primer: 5'-CGTCATTGGGTGGGTGTT-3'; reverse primer: 5'-AGCTGGGTCA CAGCCAAG-3'; TaqMan probe: 5'-FAM-TCAGCAGTGTGTCCGTGAAGCT CA-TAMRA-3'. Fold-change analysis was based on standardizing RNA levels by normalizing to glyceraldehyde-3-phosphate dehydrogenase levels in the sample.

\section{Endothelium-dependent vascular responses}

Endothelium-dependent vascular responses were measured after preparation of thoracic rat aortic rings, as reported previously. ${ }^{20}$ Briefly, cylindrical $3.0 \mathrm{~mm}$ long segments were dissected from the aorta and bathed in Krebs bicarbonate saline $\left(120 \mathrm{~mm} \mathrm{NaCl}, 5.2 \mathrm{~mm} \mathrm{KCl}, 2.4 \mathrm{mM} \mathrm{CaCl}_{2}, 1.2 \mathrm{mM} \mathrm{MgSO}_{4}, 25 \mathrm{~mm}\right.$ $\mathrm{NaHCO}_{3}, 0.03 \mathrm{~mm} \mathrm{Na}$-ethylenediaminetetraacetic acid and $11 \mathrm{~mm}$ dextrose ( $\mathrm{pH}$ 7.4) ) equilibrated with $95 \% \mathrm{O}_{2}$ and $5 \% \mathrm{CO}_{2}$, and maintained at $37^{\circ} \mathrm{C}$. The rings were suspended under $1 \mathrm{~g}$ of tension and preconstricted by adding $3 \times 10^{-7} \mathrm{M}$ norepinephrine. After the contraction force had reached a plateau, 
acetylcholine $\left(10^{-9}-10^{-5} \mathrm{M}\right.$; endothelium-dependent vasodilator $)$ or sodium nitroprusside $\left(10^{-9}-10^{-5} \mathrm{M}\right.$; endothelium-independent vasodilator) was added incrementally to the bath. The force of isometric contraction was measured using a force-displacement transducer (Model MTOB-1Z; Labo Support, Osaka, Japan). Responses to acetylcholine were expressed as the percentage of the preconstricted tension induced by norepinephrine. Nonlinear regression (GraphPad Prism5.0; GraphPad Software, La Jolla, CA, USA) was used to determine $\mathrm{pEC}_{50}$ and maximum responses $\left(R_{\max }\right)$ to acetylcholine and nitroprusside in each ring.

\section{Renal microvascular responses to step changes in renal perfusion pressure}

The effects of perfusion pressure on renal microvascular tone were examined by slight modification of the isolated perfusion technique described by van Dokkum et al. ${ }^{21}$ For perfusion of the kidneys, animals were anesthetized with sevoflurane, the left kidney was denervated by stripping all visible nerves from the renal artery, the artery was coated with a 5\% solution of phenol in ethanol, and the left renal artery was then cannulated by introducing a perfusion cannula through the mesenteric artery and across the aorta. The perfusion medium consisted of Krebs-Ringer bicarbonate buffer containing $6.5 \mathrm{~g} / 100 \mathrm{ml}$ bovine serum albumin, $5 \mathrm{~mm}$ D-glucose and a complement of amino acids. The perfusion pressure, monitored at the level of the renal artery, was altered by controlling the backpressure regulator. The renal artery was supplied with a 1-mm Transonic flow probe connected to a Transonic T206 flowmeter (Transonic Systems, Ithaca, NY, USA) to monitor renal flow. Renal perfusion pressure was maintained at $100 \mathrm{~mm} \mathrm{Hg}$. The pressure was then raised in a stepwise manner by $20-\mathrm{mm} \mathrm{Hg}$ increments to $160 \mathrm{~mm} \mathrm{Hg}$. Autoregulatory index (AI) between $100-160 \mathrm{~mm} \mathrm{Hg}$ of pressure changes was calculated by the method of Semple and de Wardener ${ }^{22}$ as follows: $\mathrm{AI}=\left[\left(\right.\right.$ renal flow $\left.(\mathrm{RF})_{2}-\mathrm{RF}_{1}\right) /$ $\left.\mathrm{RF}_{1}\right] /\left[\left(\right.\right.$ renal perfusion pressure $\left.\left.(\mathrm{RPP})_{2}-\mathrm{RPP}_{1}\right) / \mathrm{RPP}_{1}\right]$.

\section{Statistical analysis}

Values are expressed as mean \pm s.e.m. Statistical comparisons except the glomerular damage score were made using the one-factor analysis of variance with a Tukey-Kramer test for multiple comparisons. The glomerular damage score was evaluated by the Kruskal-Wallis test with the Steel-Dwass test for multiple comparisons. A $P$-value $<0.05$ denoted a statistically significant difference.

\section{RESULTS}

\section{Pathophysiological data}

Systolic blood pressure was significantly higher in DS-H rats than in DS-L rats (Table 1). A two-week treatment with telmisartan decreased systolic blood pressure in a manner similar to hydralazine. There were no differences in serum creatinine and blood urea nitrogen levels among DS-H, DS-H-Hyd and DH-H-Tel rats. Urinary protein excre- tion in DS-H rats was greater than in DS-L rats $(P<0.05)$. Urinary protein excretion was significantly decreased in both DS-H-Hyd and DH-H-Tel rats compared with DS-H rats $(P<0.05$ each), and was much lower in DH-H-Tel rats than in DS-H-Hyd rats $(P<0.05)$. Glomerular damage, which was worse in DS-H rats than in DS-L rats, also did not worsen after treatment with telmisartan compared with hydralazine (data not shown). Glomerular damage score was significantly improved in DH-H-Tel rats compared with DS-H and DS-H-Hyd rats (Table 1).

\section{Source of ROS production in glomeruli of salt-sensitive} hypertensive rats

The production of ROS was measured by dihydroethidium assay. The production of ROS was higher in the glomeruli of DS-H rats than DS-L rats (Figure 1a; $P<0.05$ ) and increased in the former group after the addition of NADPH $(3.2 \pm 0.4$-fold; $P<0.05$ vs. DS-H $(-))$. Moreover, ROS production was accelerated by the addition of L-Arg (2.5 \pm 0.4 -fold, $P<0.05$ vs. DS-H $(-))$ and decreased by the addition of NG-nitro-L-Arg methyl ester $(1.2 \pm 0.1$-fold, $P<0.05$ vs. DS-H $(-))$. There were no changes observed with the addition of succinate plus antimycin or xanthine. Furthermore, in DS-H-Hyd rats, ROS production was accelerated by the addition of L-Arg (Figurelb; $2.2 \pm 0.3$-fold, $P<0.05$ vs. DS-H-Hyd $(-))$ and diminished by incubation with BH4 (1.2 \pm 0.2 -fold, $P<0.05$ vs. DS-H-Hyd+L-Arg). However, L-Arg did not increase ROS production in DS-H-Tel rats.

\section{In situ detection of NOS coupling in glomeruli and small} arteries of salt-sensitive hypertensive rats

Figure 2 shows in situ endothelial NO and ROS images for the four groups of rats. The NO detected by DAR-4 M AM appears red (Figures 2a-d), and ROS detected by DCFH-DA appears green (Figures 2e-h). NO, but not ROS, was detected in the glomeruli and arterioles of DS-L rats. However, ROS production was increased and NO production was decreased in the glomeruli and arterioles of DS-H rats compared with DS-L rats. The production of ROS was increased and NO production was reduced in the glomeruli and arterioles of the Hyd group. Treatment with telmisartan normalized the production of NO and ROS in the glomeruli and arterioles.

\section{Western blot analysis of eNOS in glomeruli of salt-sensitive hypertensive rats}

Total eNOS was examined by standard SDS-PAGE. Dimer/monomer eNOSs were examined by low-temperature SDS-PAGE. Total eNOS

Table 1 Pathophysiological data

\begin{tabular}{|c|c|c|c|c|}
\hline & $D S-L$ & $D S-H$ & $D S-H-H y d$ & $D S-H-T e l$ \\
\hline \multicolumn{5}{|l|}{ Physiological data $(n=15)$} \\
\hline Body weight (g) & $433 \pm 10$ & $386 \pm 7^{*}$ & $402 \pm 9$ & $402 \pm 8$ \\
\hline Systolic blood pressure $(\mathrm{mm} \mathrm{Hg})$ & $114 \pm 2$ & $190 \pm 3^{*}$ & $136 \pm 3^{* *}$ & $134 \pm 3^{* *}$ \\
\hline Serum creatinine (mg $100 \mathrm{ml}^{-1}$ ) & $0.27 \pm 0.01$ & $0.37 \pm 0.02 *$ & $0.35 \pm 0.02$ & $0.38 \pm 0.02$ \\
\hline Blood urea nitrogen (mg $100 \mathrm{ml}^{-1}$ ) & $18.9 \pm 1.0$ & $24.2 \pm 1.3^{*}$ & $22.9 \pm 1.8$ & $22.3 \pm 1.1$ \\
\hline Urinary protein excretion (mg per day) & $27.0 \pm 2.9$ & $106.4 \pm 6.9^{*}$ & $70.7 \pm 5.7^{* *}$ & $52.1 \pm 3.3^{* * * * * *}$ \\
\hline \multicolumn{5}{|l|}{ Histology $(n=5)$} \\
\hline Glomerular damage (score 0-4) & $0.18 \pm 0.02$ & $1.35 \pm 0.14^{*}$ & $1.10 \pm 0.06^{* *}$ & $0.79 \pm 0.08^{* *, * * *}$ \\
\hline
\end{tabular}

Abbreviations: DS-H, untreated DS rats; DS-H-Hyd, DS rats treated with hydralazine; DS-L, DS normotensive rats fed a $0.9 \%$ salt diet; DS-H-Tel, DS rats treated with telmisartan.

Data are expressed as mean \pm s.e.m.

${ }^{\star} P<0.05$ vs. DS-L.

${ }^{*} P<0.05$ vs. DS-H.

${ }_{* * *} P<0.05$ vs. DS-H-Hyd. 


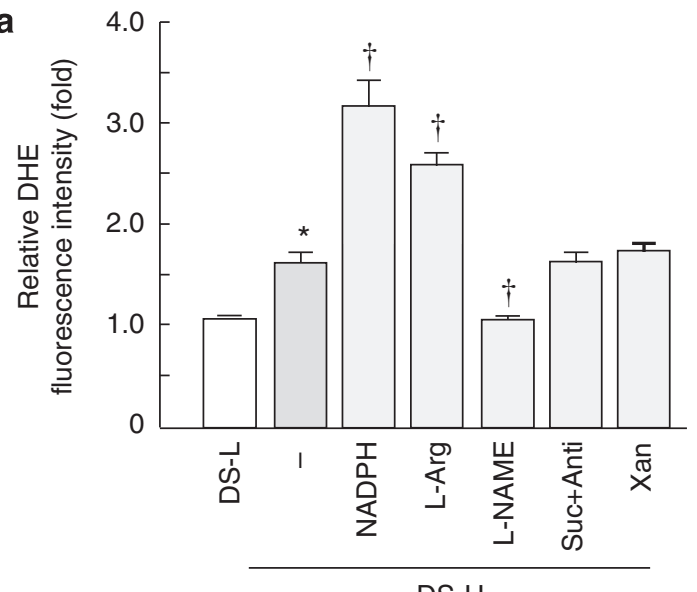

b

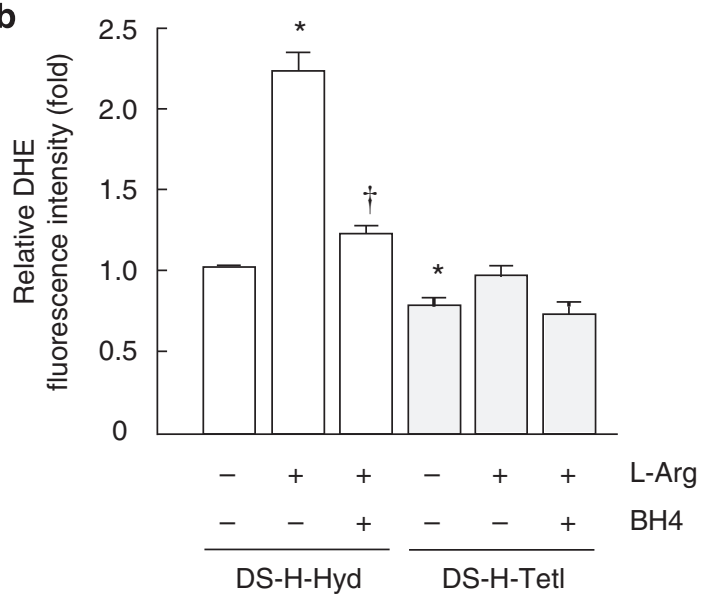

Figure 1 Source of reactive oxygen species (ROS) production. ROS production was measured by dihydroethidium (DHE) assay. (a) L-Arg, L-arginine; Suc+Anti, succinate+antimycin; Xan, xanthine. $n=5$ in each group. ${ }^{*} P<0.05$ vs. DS-L (DS normotensive rats fed a $0.9 \%$ salt diet). ${ }^{\dagger} P<0.05$ vs. DS-H (untreated DS rats) (-). (b) $n=5$ in each group. ${ }^{*} P<0.05$ vs. DS-H-Hyd (DS rats treated with hydralazine) $(-) .{ }^{\dagger} P<0.05$ vs. DS-H-Hyd L-Arg (+). DS, Dahl salt-sensitive hypertensive.

production was similar in the four groups (Figure 3a). However, the ratio of dimer to monomer eNOS was lower in DS-H rats than DS-L rats (Figure $3 \mathrm{~b}, P<0.05)$. Treatment with telmisartan $(P<0.05 v s$. DS$\mathrm{H}-\mathrm{Hyd})$, but not hydralazine, ameliorated the decrease of the eNOS dimer/monomer ratio.

\section{Biopterin production and concentration in salt-sensitive hypertensive rats}

Tetrahydrobiopterin (BH4) is an essential cofactor required for the production of NO by each of the NOS isoforms. Synthesis of BH4 involves a multistep process in which GTPCH1 is the rate-limiting enzyme required for the initial step in conversion of guanosine- $5^{\prime}$ triphosphate to $\mathrm{BH} 4$. The mRNA expression of GTPCH1, as measured by real-time PCR (Figure 3c), was increased in DS-H, DS-H-Hyd and DS-H-Tel rats compared with DS-L rats, but there were no differences between the three groups. Serum BH4 levels were decreased in DS-H and DS-H-Hyd rats, but preserved in DS-H-Tel rats (Figure $3 \mathrm{~d}$ ). However, serum levels of $\mathrm{BH} 2$, which is the oxidized form of $\mathrm{BH} 4$, were increased in DS-H and DS-H-Hyd rats, but preserved in DS-HTel rats (Figure 3e).
Aortic artery endothelial function in salt-sensitive hypertensive rats Endothelium-dependent relaxation of aortic artery rings in response to acetylcholine was significantly depressed in DS-H rats compared with DS-L rats (Figure 4a). Acetylcholine caused concentrationdependent vasorelaxations in preconstricted control rings from DS-L rats $\left(\mathrm{pEC}_{50}=7.2 \pm 0.1 ; R_{\max }=82 \pm 4 \% ; n=5\right)$. In contrast, both the sensitivity and maximum relaxations to acetylcholine enhanced in collared rings were depressed in rings from DS-L rats $\left(\mathrm{pEC}_{50}=6.5 \pm 0.2 ; R_{\max }=27 \pm 3 \% ; n=5 ; P<0.05\right)$. Telmisartan and hydralazine significantly $(P<0.05)$ improved both sensitivity $\left(\mathrm{pEC}_{50}=6.9 \pm 0.1\right.$ and $6.9 \pm 0.1$, respectively; $n=6$ each) and maximum relaxation $\left(R_{\max }=60 \pm 1\right.$ and $37 \pm 2 \%$, respectively; $n=5$ each) to acetylcholine in aortic artery ring segments compared with the rings from DS-H rats. Regarding maximum relaxation, telmisartan treatment afforded more improvement $(P<0.05)$ than hydralazine treatment. However, endothelium-independent relaxation of the aortic artery rings in response to sodium nitroprusside did not differ in any group of rats (Figure $4 \mathrm{~b}$ ). To determine whether endothelial cells were histologically damaged, expression of rat endothelial cell antigen 1 in the isolated aortic artery was examined. Endothelial cells were preserved in DS-H rats (data not shown).

Renal flow autoregulation in salt-sensitive hypertensive rats Alterations in renal flow during step changes in renal perfusion pressure are shown in Figure 5. The capacity to autoregulate the steady-state renal flow in response to step changes was decreased in DS-H rats (Figure 5b) compared with DS-L rats (Figure 5a), as indicated by the difference in the calculated AI between 100$160 \mathrm{~mm} \mathrm{Hg}$ of pressure changes $(\mathrm{AI}=0.56 \pm 0.07$ and $0.22 \pm 0.03$, respectively, $P<0.05)$. The renal flow autoregulation in response to step changes in perfusion pressure was well improved in DS-H-Tel rats (Figure 5d; $\mathrm{AI}=0.34 \pm 0.05$ ) but not in DS-H-Hyd rats (Figure 5c; $\mathrm{AI}=0.64 \pm 0.08, P<0.05$ vs. DS-H-Tel).

Purinergic receptor expression in salt-sensitive hypertensive rats The mRNA expression levels of P2rx1 in renal cortex were examined by quantitative real-time PCR. The P2rxl mRNA expression could be detected in the renal cortex, and the expression was decreased in hypertensive rat glomeruli (Figure 6; $0.75 \pm 0.06$-fold in DS-H compared with DS-L, $P<0.05)$. Telmisartan treatment restored the P2rx1 expression to control levels $(0.96 \pm 0.12$-fold $)$.

\section{DISCUSSION}

In this study, we explored whether uncoupled eNOS parallel with endothelial dysfunction and deterioration of renal autoregulation were involved in the progression of hypertensive renal disease. Our results demonstrated that NO/ROS imbalance, which is indicative of endothelial dysfunction, exists in DS rat glomerular and pre-glomerular arterioles, and that telmisartan has beneficial effects against eNOS uncoupling, endothelial dysfunction and loss of renal autoregulation in a Dahl rat model for salt-sensitive hypertensive renal disease.

We reported previously that NADPH oxidase and uncoupled NOS are major sources of glomerular superoxide in streptozotocin-induced rats with diabetic nephropathy, ${ }^{17}$ and NOS uncoupling has been reported to occur in the renal medulla of DS rats. ${ }^{23}$ In this study, we demonstrated that uncoupled eNOS also occurs in the glomeruli of DS rats. The eNOS uncoupling was found to participate not only in kidney disease, but also in arteriosclerosis and endothelial dysfunction in large blood vessels of diabetics. ${ }^{8}$ Thus, eNOS uncoupling seems to be a common mechanism of endothelial dysfunction, leading to reduced NO production and increased ROS production in the blood vessel wall. 

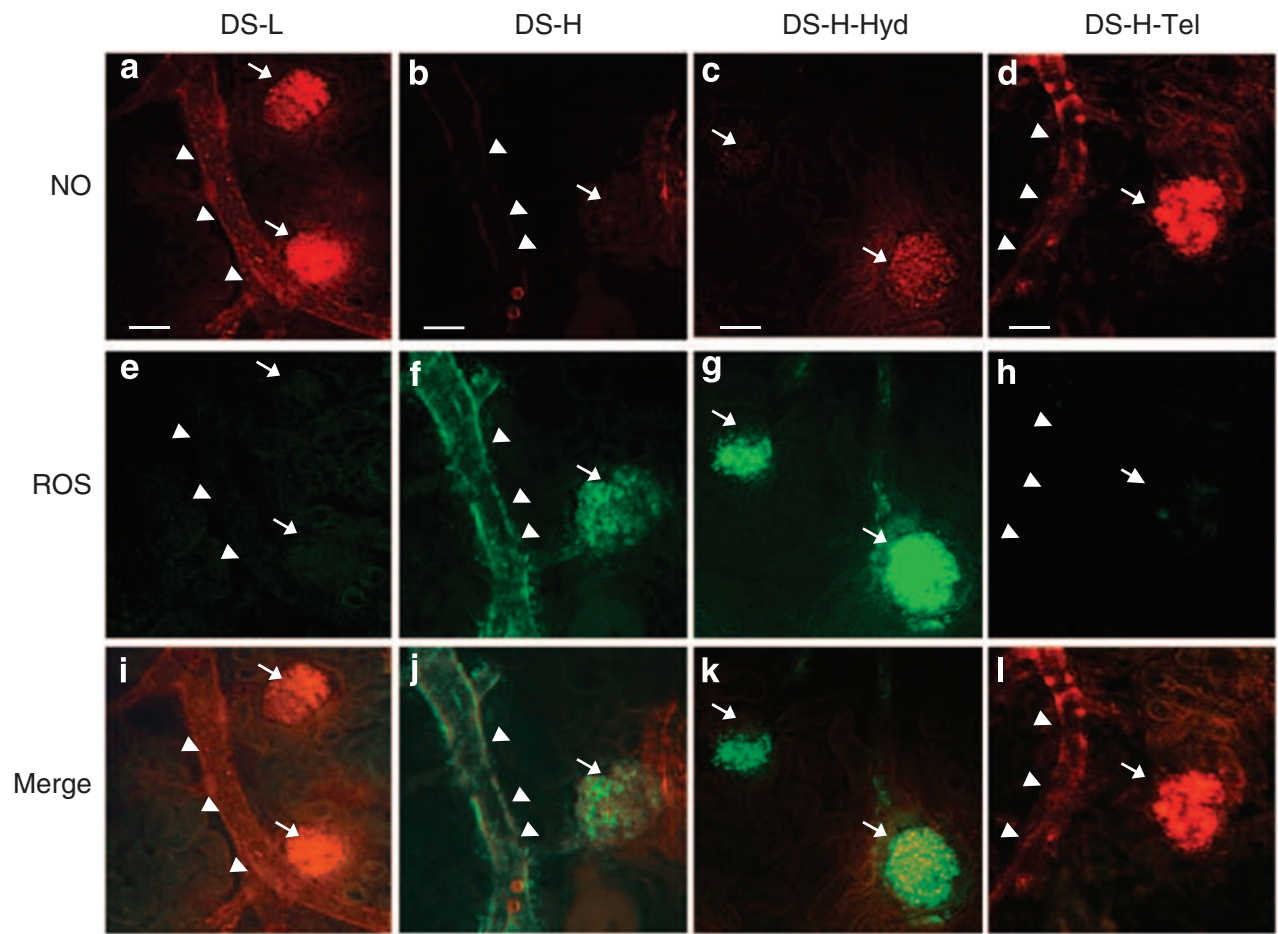

Figure 2 In situ detection of nitric oxide (NO) and reactive oxygen species (ROS). (a-d) Upper panel: NO detected by diaminorhodamine-4 $\mathrm{m}$ acetoxymethyl ester (DAR-4 M AM). (e-h) Middle panel: ROS detected by dichlorodihydrofluorescein (DCFH). (i-l) Lower panel: merged images of NO with ROS. Arrow: glomeruli; arrowheads: small lobar artery. Data are representative of five independent experiments. Bar $=100 \mu \mathrm{m}$.

a

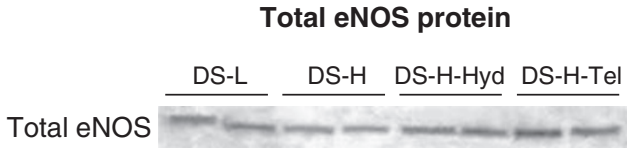

b
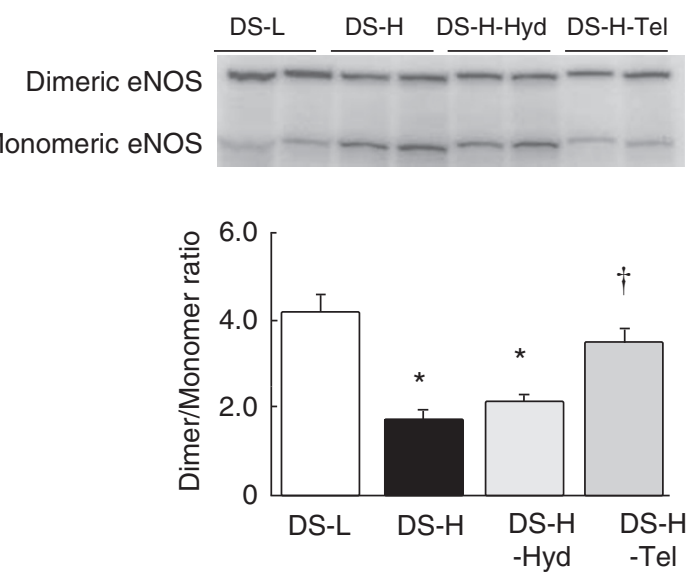
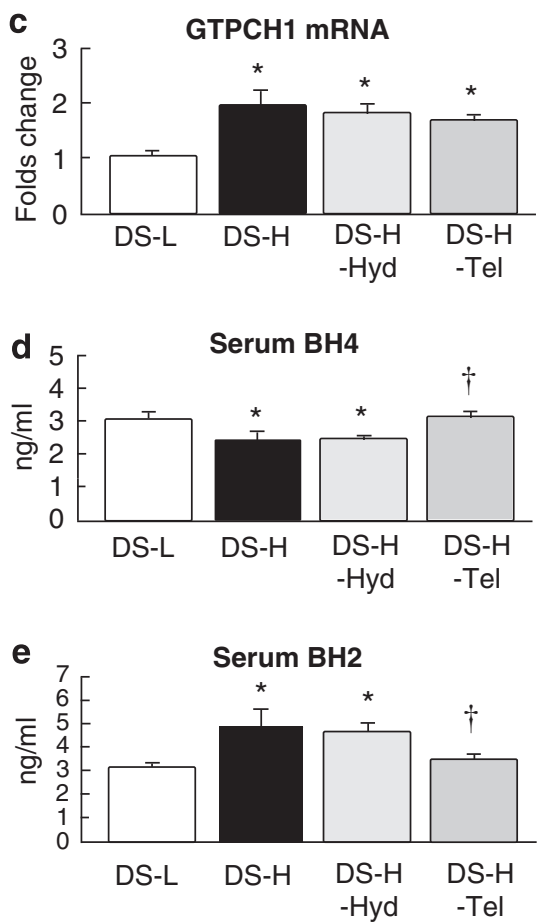

Figure 3 Western blot analysis of endothelial nitric oxide synthase (eNOS) and biopterin production. (a) Total eNOS was examined by normal SDS-PAGE. (b) Dimer/monomer eNOS were examined by low temperature SDS-PAGE. $n=6$ in each group. ${ }^{*} P<0.05$ vs. DS-L (DS normotensive rats fed a $0.9 \%$ salt diet), ${ }^{\dagger} \mathrm{P}<0.05$ vs. DS-H-Hyd (DS rats treated with hydralazine). (c) Real-time PCR for guanosine-5'-triphosphate cyclohydrolase 1 (GTPCH1) mRNA expression. $n=5$ in each group. ${ }^{*} P<0.05$ vs. DS-L. (d) Serum tetrahydrobiopterin (BH4) concentration. (e) Dihydrobiopterin (BH2) concentration. $n=5$ in each group. ${ }^{*} P<0.05$ vs. DS-L, ${ }^{\dagger} P<0.05$ vs. DS-H-Hyd. DS, Dahl salt-sensitive hypertensive. 
a Enodothelium dependent vasodilation

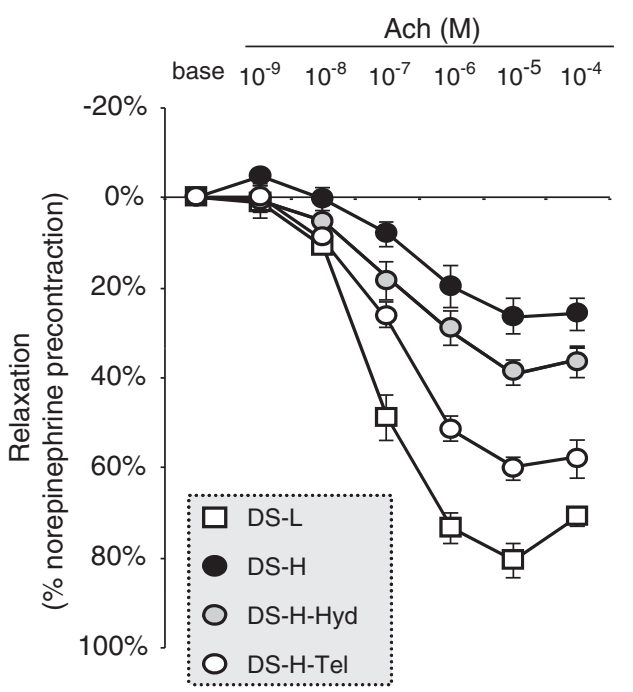

b Enodothelium independent vasodilation

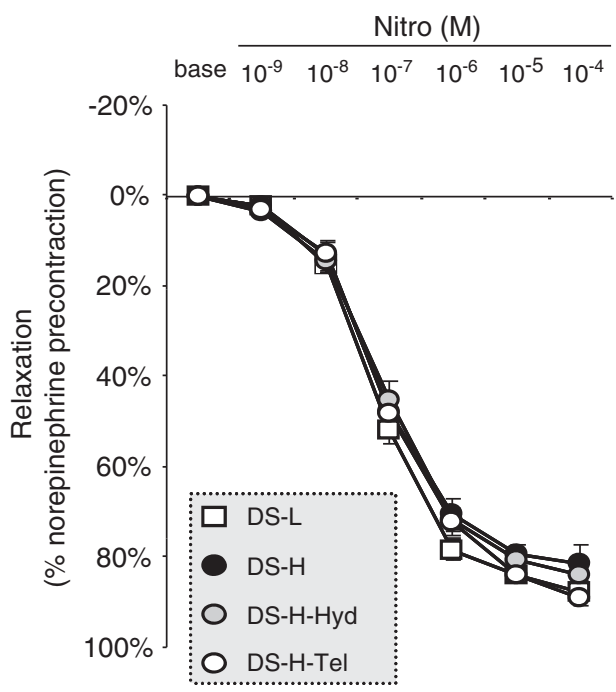

Figure 4 Endothelial function of aortic artery. (a) Endothelium-dependent vasodilation examined by acetylcholine. (b) Endothelium-independent vasodilation examined by nitroprusside. $n=5$ in each group.

In DS rats, high salt intake induced the activation of NADPH oxidase , ${ }^{24}$ and in another study, such activation increased ROS production, with subsequent endothelial dysfunction. ${ }^{25}$ The production of ROS by NADPH oxidase oxidized $\mathrm{BH} 4$ to $\mathrm{BH} 2$ and caused eNOS uncoupling. In addition, ROS produced by NADPH oxidase reacted with NO immediately and produced peroxynitrite. ${ }^{26}$ Reaction of this peroxynitrite with the protein promotes nitrotyrosine formation of the protein. Nitrotyrosine formation of eNOS may obstruct eNOS dimers. In addition, the resolution of GTPCH1, which is an enzyme involved in the production of $\mathrm{BH} 4$, is promoted by proteasome-dependent degradation under the presence of peroxynitrite. ${ }^{27}$ Consequently, reduced production of $\mathrm{BH} 4$ may induce eNOS uncoupling.

Treatment with ARB clearly improved eNOS uncoupling. The mechanism of this action of ARB is thought to be mainly a reduction of ROS production by attenuation of glomerular hypertension and inhibition of NADPH oxidase. There is a close link between NADPH
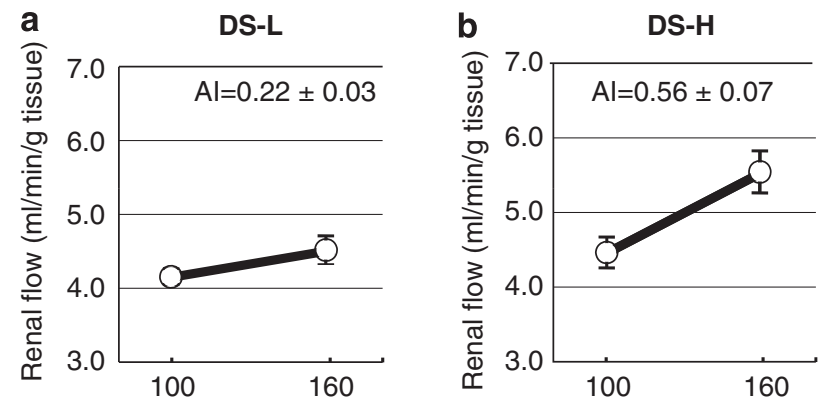

Renal perfusion pressure $(\mathrm{mmHg})$

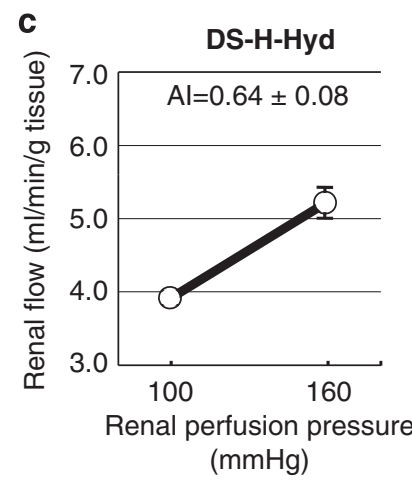

Renal perfusion pressure $(\mathrm{mmHg})$

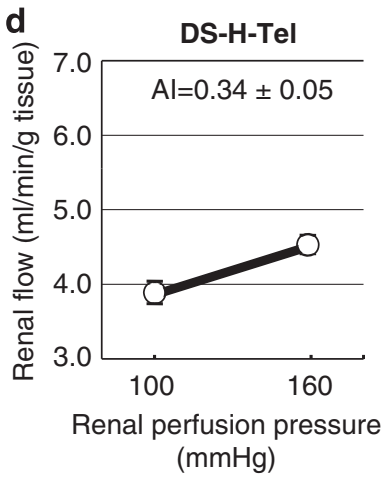

Figure 5 Renal flow autoregulation in DS-L (DS normotensive rats fed a $0.9 \%$ salt diet) (a), DS-H (untreated DS rats) (b), DS-H-Hyd (DS rats treated with hydralazine) (c) and DS-H-Tel (DS rats treated with telmisartan) (d). $n=6$ in each group. Autoregulatory index $(\mathrm{Al})=\left[\left(\mathrm{RF}_{2}-\mathrm{RF}_{1}\right) / \mathrm{RF}_{1}\right] /\left[\left(\mathrm{RPP}_{2}-\right.\right.$ $\left.\mathrm{RPP}_{1}\right) / \mathrm{RPP}_{1}$ ]. DS, Dahl salt-sensitive hypertensive; $R F$, renal flow; $R P P$, renal perfusion pressure.

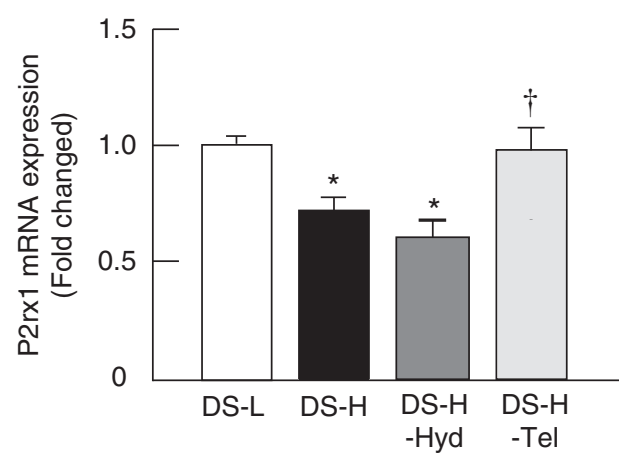

Figure 6 Effects of telmisartan on renal P2rx 1 mRNA expression in Dahl salt-sensitive hypertensive (DS) rats. Real-time PCR for P2rx1 mRNA expression. $n=6$ in each group. ${ }^{*} P<0.05$ vs. DS-L (DS normotensive rats fed a $0.9 \%$ salt diet), ${ }^{\dagger} P<0.05$ vs. DS-H-Hyd (DS rats treated with hydralazine).

oxidase activation and NOS uncoupling, ${ }^{28}$ and it has been reported that restraint of NADPH oxidase improved NOS uncoupling. ${ }^{29}$ In DS rats, NADPH oxidase-induced superoxide production may trigger eNOS uncoupling, leading to impaired NO signaling and to endothelial dysfunction. Angiotensin receptor blockade (ARB) was demonstrated to decrease NADPH oxidase activity. ${ }^{18}$ Therefore, reduction of NADPH oxidase activity by ARB seems the most efficient method to improve endothelial function through NOS recoupling. However, the 
abnormal feature in DS rats is characterized by the dysfunction of NO system. Barton et al..$^{30}$ reported that DS rats failed to increase renal NOS activity in response to salt loading. The ARB treatment may improve the NOS activity in response to salt loading.

The inability of eNOS to produce NO on a regular basis leads to an imbalance between ROS and NO, and endothelial dysfunction. In this study, we measured endothelium-dependent relaxation of the aortic artery rings in response to acetylcholine. The relaxation response significantly fell in DS rats with disproportionate NO/ROS production. In addition, we measured the ability of autoregulatory responses, which was significantly lower in DS rat kidneys. The autoregulatory response is reported to be significantly decreased in DS rats ${ }^{31}$ and remnant kidney rats, a model of progressive renal damage. ${ }^{32}$ The renal autoregulatory response is coordinated by two mechanisms: myogenic response and tubulo-glomerular feedback. ${ }^{33}$ On the basis of the results of our experiments in DS rats, ARB seems to improve the renal autoregulatory response in parallel with endothelial function. The endothelium has a central role in early functional adaptations to hypertension. Pressure-induced myogenic constriction is enhanced due to the augmented release of endothelium-derived constrictor factors that modulate arteriolar smooth muscle sensitivity to $\mathrm{Ca}^{2+} .34$ In contrast, flow/shear stress-induced dilation of arterioles is reduced in hypertension, due to the impaired mediation of the response by NO. $^{35}$ It has also been proposed that high salt intake reduces endothelium-dependent dilation of mouse arterioles by enhancing the release of ROS from NOS, which then interferes with the synthesis and/or action of endothelium-derived mediators. ${ }^{36}$ Treatment with ARB might maintain and improve endothelial function by reducing superoxide production, which could lead to inhibition of vascular constrictive changes due to the improved mediation of the response by NO and consequently may maintain a normal pressure-induced myogenic constriction.

In general, angiotensin II promotes the autoregulatory response reaction in normal rats by increasing tubulo-glomerular feedback. ${ }^{37,38}$ So, ARB may dampen the strength of tubulo-glomerular feedback helping to restore the normal GFR response. ${ }^{39}$ Enhanced tubuloglomerular feedback responses may be the result of an angiotensin IIinduced reduction in local levels of nitric oxide. ${ }^{40}$ Tubulo-glomerular feedback responses have been reported to be enhanced in spontaneously hypertensive rats, ${ }^{41}$ but there are few reports regarding to tubulo-glomerular feedback in DS rats. It is well accepted that DS rats on high-salt diet exhibit a suppressed systemic RAS (renninangiotensin system) accompanied with inappropriately activated renal RAS. ${ }^{42}$ Indeed, there is a possibility that the tubuloglomerular feedback response in the DS rats are reduced similar to deoxycorticosterone acetate (DOCA)- salt hypertensive rat or Goldblatt hypertensive rats. ${ }^{43}$ So, ARB might have little effects on the renal autoregulatory mechanism via tubulo-glomerular feedback in DS rats.

We have shown that the renal autoregulation in the DS rat was impaired. Conclusive identification of the signaling molecules that mediate autoregulation remains controversial, but it does seem clear that extracellular ATP and P2 purine receptor activation is required for autoregulatory control of renal hemodynamics. ${ }^{44,45}$ Indeed, ATP is released from many different cell types in response to stretch, osmotic stimuli ${ }^{46}$ and activation of P2rxl receptors, which is an essential step in pressure-mediated afferent arteriolar vasoconstriction. Blockade of $\mathrm{P} 2 \mathrm{rx} 1$ receptors or deletion of $\mathrm{P} 2 \mathrm{rx} 1$ receptors in knockout mice eliminates pressure-mediated afferent arteriolar contraction. ${ }^{47}$ In hypertensive renal disease, pathological changes arise in small arteries in the early stage. ${ }^{48}$ So, this ATP-P2X axis might be damaged, which could subsequently lead to down regulation of pressure sensory ability and cause glomerular hypertensive injury. Actually, mRNA expression levels of P2rx1 in the renal cortex of DS rats were decreased, which seem to be parallel with impairment of renal autoregulation. Telmisartan effectively prevented the deterioration of renal autoregulation, which might be due to the preservation of P2rx1.

The relationship between endothelial function and renal autoregulational ability is not clear from the results of this study. Decreased endothelial function might induce the failure of autoregulation. We demonstrated that treatment with telmisartan improved both functions in DS rat kidney, but there are no data to support the hypothesis that the decreased endothelial function lies behind the decreased autoregulation. Similarly, whether a causal relation exists between improved autoregulation and a decrease in renal damage cannot be determined from this study. It is very difficult to design experiments that directly address these important questions, but this is what is needed if we are to gain new insight into these questions in the future.

In summary, we detected glomerular ROS/NO imbalance and impairment of renal autoregulatory response in rats with salt-induced hypertension. We also showed that correction of the ROS/NO disproportion and endothelial dysfunction were improved by ARB treatment.

\section{CONFLICT OF INTEREST}

The authors declare no conflict of interest.

\section{ACKNOWLEDGEMENTS}

We thank M Ono and S Tsujita for excellent technical assistance. This study was supported by grants from KAKENHI (No. 19590969) to NK and the Salt Science Research Foundation (No. 0731) to MS. Telmisartan was kindly supplied by Astellas Pharma (Tokyo, Japan).

1 Klag MJ, Whelton PK, Randall BL, Neaton JD, Brancati FL, Ford CE, Shulman NB, Stamler J. Blood pressure and end-stage renal disease in men. N Engl J Med 1996; 334: 13-18.

2 Tozawa M, Iseki K, Iseki C, Kinjo K, Ikemiya Y, Takishita S. Blood pressure predicts risk of developing end-stage renal disease in men and women. Hypertension 2003; 41: 1341-1345.

3 The GISEN Group (Gruppo Italiano di Studi Epidemiologici in Nefrologia). Randomised placebo-controlled trial of effect of ramipril on decline in glomerular filtration rate and risk of terminal renal failure in proteinuric non-diabetic nephropathy. Lancet 1997; 349: 1857-1863.

4 Walker WG, Neaton JD, Cutler JA, Neuwirth R, Cohen JD. Renal function change in hypertensive members of the Multiple Risk Factor Intervention Trial. Racial and treatment effects. The MRFIT Research Group. JAMA 1992; 268: 3085-3091.

5 Feld LG, Van Liew JB, Galaske RG, Boylan JW. Selectivity of renal injury and proteinuria in the spontaneously hypertensive rat. Kidney Int 1977; 12: 332-343.

6 Cai H, Harrison DG. Endothelial dysfunction in cardiovascular diseases: the role of oxidant stress. Circ Res 2000; 87: 840-844.

7 Stuehr D, Pou S, Rosen GM. Oxygen reduction by nitric-oxide synthases. J Biol Chem 2001; 276: 14533-14536.

8 Oelze M, Mollnau H, Hoffmann N, Warnholtz A, Bodenschatz M, Smolenski A, Walter U, Skatchkov M, Meinertz T, Munzel T. Vasodilator-stimulated phosphoprotein serine 239 phosphorylation as a sensitive monitor of defective nitric oxide/cGMP signaling and endothelial dysfunction. Circ Res 2000; 87: 999-1005.

9 Hink U, Li H, Mollnau H, Oelze M, Matheis E, Hartmann M, Skatchkov M, Thaiss F, Stahl RA, Warnholtz A, Meinertz T, Griendling K, Harrison DG, Forstermann U, Munzel T. Mechanisms underlying endothelial dysfunction in diabetes mellitus. Circ Res 2001; 88: E14-E22.

10 Xia Y, Tsai AL, Berka V, Zweier JL. Superoxide generation from endothelial nitric-oxide synthase. A Ca2+/calmodulin-dependent and tetrahydrobiopterin regulatory process. J Biol Chem 1998; 273: 25804-25808.

11 Vasquez-Vivar J, Kalyanaraman B, Martasek P, Hogg N, Masters BS, Karoui H, Tordo P, Pritchard Jr KA. Superoxide generation by endothelial nitric oxide synthase: the influence of cofactors. Proc Natl Acad Sci USA 1998; 95: 9220-9225.

12 Alp NJ, Channon KM. Regulation of endothelial nitric oxide synthase by tetrahydrobiopterin in vascular disease. Arterioscler Thromb Vasc Biol 2004; 24: 413-420.

13 Persson PB. Renal blood flow autoregulation in blood pressure control. Curr Opin Nephrol Hypertens 2002; 11: 67-72. 
14 Inscho EW, Carmines PK, Cook AK, Navar LG. Afferent arteriolar responsiveness to altered perfusion pressure in renal hypertension. Hypertension 1990; 15: 748-752.

15 Inscho EW, Imig JD, Deichmann PC, Cook AK. Candesartan cilexetil protects against loss of autoregulatory efficiency in angiotensin II-infused rats. J Am Soc Nephrol 1999; 10(Suppl 11): S178-S183.

16 Haruna Y, Morita Y, Yada T, Satoh M, Fox DA, Kashihara N. Fluvastatin reverses endothelial dysfunction and increased vascular oxidative stress in rat adjuvant-induced arthritis. Arthritis Rheum 2007; 56: 1827-1835.

17 Satoh M, Fujimoto S, Haruna Y, Arakawa S, Horike H, Komai N, Sasaki T, Tsujioka K, Makino $\mathrm{H}$, Kashihara N. NAD(P)H oxidase and uncoupled nitric oxide synthase are major sources of glomerular superoxide in rats with experimental diabetic nephropathy. Am J Physiol Renal Physiol 2005; 288: F1144-F1152.

18 Fujimoto S, Satoh M, Horike H, Hatta H, Haruna Y, Kobayashi S, Namikoshi T, Arakawa $\mathrm{S}$, Tomita N, Kashihara N. Olmesartan ameliorates progressive glomerular injury in subtotal nephrectomized rats through suppression of superoxide production. Hypertens Res 2008; 31: 305-313.

19 Satoh M, Fujimoto S, Arakawa S, Yada T, Namikoshi T, Haruna Y, Horike H, Sasaki T, Kashihara N. Angiotensin II type 1 receptor blocker ameliorates uncoupled endothelial nitric oxide synthase in rats with experimental diabetic nephropathy. Nephrol Dial Transplant 2008; 23: 3806-3813.

20 Namikoshi T, Tomita N, Satoh M, Haruna Y, Kobayashi S, Komai N, Sasaki T, Kashihara $\mathrm{N}$. Olmesartan ameliorates renovascular injury and oxidative stress in Zucker obese rats enhanced by dietary protein. Am J Hypertens 2007; 20: 1085-1091.

21 van Dokkum RP, Sun CW, Provoost AP, Jacob HJ, Roman RJ. Altered renal hemodynamics and impaired myogenic responses in the fawn-hooded rat. Am J Physiol 1999; 276: R855-R863.

22 Semple SJ, De Wardener HE. Effect of increased renal venous pressure on circulatory autoregulation of isolated dog kidneys. Circ Res 1959; 7: 643-648.

23 Taylor NE, Maier KG, Roman RJ, Cowley Jr AW. NO synthase uncoupling in the kidney of Dahl S rats: role of dihydrobiopterin. Hypertension 2006; 48: 1066-1071.

24 Fujii S, Zhang L, Igarashi J, Kosaka H. L-arginine reverses p47phox and gp91phox expression induced by high salt in Dahl rats. Hypertension 2003; 42: 1014-1020.

25 Ray R, Shah AM. NADPH oxidase and endothelial cell function. Clin Sci (Lond) 2005; 109: 217-226.

26 Squadrito GL, Pryor WA. The formation of peroxynitrite in vivo from nitric oxide and superoxide. Chem Biol Interact 1995; 96: 203-206.

27 Xu J, Wu Y, Song P, Zhang M, Wang S, Zou MH. Proteasome-dependent degradation of guanosine 5'-triphosphate cyclohydrolase I causes tetrahydrobiopterin deficiency in diabetes mellitus. Circulation 2007; 116: 944-953.

$28 \mathrm{Xu} \mathrm{J}$, Xie Z, Reece R, Pimental D, Zou MH. Uncoupling of endothelial nitric oxidase synthase by hypochlorous acid: role of $\mathrm{NAD}(\mathrm{P}) \mathrm{H}$ oxidase-derived superoxide and peroxynitrite. Arterioscler Thromb Vasc Biol 2006; 26: 2688-2695.

29 Oelze M, Daiber A, Brandes RP, Hortmann M, Wenzel P, Hink U, Schulz E, Mollnau H, von Sandersleben A, Kleschyov AL, Mulsch A, Li H, Forstermann U, Munzel T. Nebivolol inhibits superoxide formation by NADPH oxidase and endothelial dysfunction in angiotensin II-treated rats. Hypertension 2006; 48: 677-684.

30 Barton M, Vos I, Shaw S, Boer P, D'Uscio LV, Grone HJ, Rabelink TJ, Lattmann T, Moreau P, Luscher TF. Dysfunctional renal nitric oxide synthase as a determinant of salt-sensitive hypertension: mechanisms of renal artery endothelial dysfunction and role of endothelin for vascular hypertrophyandGlomerulosclerosis. J Am Soc Nephrol 2000; 11: 835-845.

31 Karlsen FM, Andersen CB, Leyssac PP, Holstein-Rathlou NH. Dynamic autoregulation and renal injury in Dahl rats. Hypertension 1997; 30: 975-983.

32 Bidani AK, Hacioglu R, Abu-Amarah I, Williamson GA, Loutzenhiser R, Griffin KA. 'Step' vs'dynamic' autoregulation: implications for susceptibility to hypertensive injury. Am J Physiol Renal Physiol 2003; 285: F113-F120.

33 Cupples WA, Braam B. Assessment of renal autoregulation. Am J Physiol Renal Physiol 2007; 292: F1105-F1123.

34 Ungvari Z, Koller A. Selected contribution: NO released to flow reduces myogenic tone of skeletal muscle arterioles by decreasing smooth muscle $\mathrm{Ca}(2+)$ sensitivity. J Appl Physiol 2001; 91: 522-527 discussion 504-525.

35 Huang A, Sun D, Kaley G, Koller A. Superoxide released to high intra-arteriolar pressure reduces nitric oxide-mediated shear stress- and agonist-induced dilations. Circ Res 1998; 83: 960-965.

36 Nurkiewicz TR, Boegehold MA. High salt intake reduces endothelium-dependent dilation of mouse arterioles via superoxide anion generated from nitric oxide synthase. Am J Physiol Regul Integr Comp Physiol 2007; 292: R1550-R1556.

37 Rahgozar M, Guan Z, Matthias A, Gobe GC, Endre ZH. Angiotensin II facilitates autoregulation in the perfused mouse kidney: An optimized in vitro model for assessment of renal vascular and tubular function. Nephrology (Carlton) 2004; 9: 288-296.

38 Guan Z, Willgoss DA, Matthias A, Manley SW, Crozier S, Gobe G, Endre ZH. Facilitation of renal autoregulation by angiotensin II is mediated through modulation of nitric oxide. Acta Physiol Scand 2003; 179: 189-201.

39 Mitchell KD, Navar LG. Enhanced tubuloglomerular feedback during peritubular infusions of angiotensins I and II. Am J Physiol 1988; 255: F383-F390.

40 Ichihara A, Hayashi M, Koura Y, Tada Y, Sugaya T, Hirota N, Saruta T. Blunted tubuloglomerular feedback by absence of angiotensin type $1 \mathrm{~A}$ receptor involves neuronal NOS. Hypertension 2002; 40: 934-939.

41 Leyssac PP, Holstein-Rathlou NH. Tubulo-glomerular feedback response: enhancement in adult spontaneously hypertensive rats and effects of anaesthetics. Pflugers Arch 1989; 413: 267-272.

42 Rapp JP, Tan SY, Margolius HS. Plasma mineralocorticoids, plasma renin, and urinary kallikrein in salt-sensitive and salt-resistant rats. Endocr Res Commun 1978; 5: 35-41.

43 Muller-Suur R, Gutsche HU, Samwer KF, Oelkers W, Hierholzer K. Tubuloglomerular feedback in rat kidneys of different renin contents. Pflugers Arch 1975; 359: 33-56.

44 Schnermann J, Levine DZ. Paracrine factors in tubuloglomerular feedback: adenosine, ATP, and nitric oxide. Annu Rev Physiol 2003; 65: 501-529.

45 Inscho EW. P2 receptors in regulation of renal microvascular function. Am J Physiol Renal Physiol 2001; 280: F927-F944.

46 Schwiebert EM. ATP release mechanisms, ATP receptors and purinergic signalling along the nephron. Clin Exp Pharmacol Physiol 2001; 28: 340-350.

47 Inscho EW, Cook AK, Imig JD, Vial C, Evans RJ. Physiological role for P2X1 receptors in renal microvascular autoregulatory behavior. J Clin Invest 2003; 112: 1895-1905.

48 Mulvany MJ. Small artery remodelling in hypertension: causes, consequences and therapeutic implications. Med Biol Eng Comput 2008; 46: 461-467. 